\title{
Diffraction mechanism of five-port transmission gratings
}

\author{
Chenhao GAO, Bo WANG* ${ }^{*}$ Kunhua WEN, Ziming MENG, Qu WANG, \\ Zhaogang NIE, Xiangunn XING, Li CHEN, Liang LEI, JinYun ZHOU
}

School of Physics and Optoelectronic Engineering, Guangdong University of Technology, Guangzhou 510006, China

*Corresponding author: wangb_wsx@yeah.net

\begin{abstract}
This paper designs a five-port transmission grating under normal incidence. Rigorous coupled-wave approach is used to optimize the grating parameters. The energy of the grating is mainly dispersed to the 0 th, \pm 1 st and \pm 2 nd orders. The efficiency of each diffraction order under both polarizations is close to $20 \%$. The modal method is used to describe the propagation mechanism of the two polarized lights in the grating, and the diffraction behavior of the grating is analyzed. In addition, the grating has a wide range of incident characteristics and a large process tolerance. Therefore, this five-port beam splitter with a connecting layer will be a good polarization-independent beam splitting device.
\end{abstract}

Keywords: $1 \times 5$ splitting, single-groove grating, normal incidence.

\section{Introduction}

Grating [ $\underline{1}-\underline{6}]$ is an important optical device. According to its basic characteristics of polarization, phase, beam splitting and dispersion [ㄱ-10], the grating can realize coupling, phase matching, splitting and filtering [11 $-\underline{14}]$. Moreover, based on integration with other devices, a grating beam splitter, a filter, and a wavelength division multiplexer can be fabricated [15-20]. Beam splitters have important application value in the field of optical communication [21, 22]. With the continuous improvement of lithography level, multi-channel grating beam splitters have been proposed. CAO et al. designed an efficient single-port fused-silica grating [23]. FeNG et al. first proposed a single-layer dielectric three-port transmissive grating [24]. XIANG et al. proposed a five-port transmission grating with single-groove structure under TE-polarized light [드] . Compared with Ref. [25] , our design not only improves the efficiency of TE polarization but also calculates the beam splitting of the grating under TM polarization.

The numerical methods for designing grating devices are mainly the rigorous coupled-wave method [26], finite element method, time domain finite difference method and modal method [27]. The method used in this design is a rigorous coupled-wave 
method and modal method. Unlike the rigorous coupled-wave approach, the electromagnetic field of the grating in the modal method is not a Fourier series expansion but an eigenmode expansion. Combined with the periodic characteristics of the grating, the modal method analyzes the modality of the grating to obtain the effective refractive index of each mode in the grating region, and the energy coupling of the modal and diffraction orders of the grating are investigated. In this design, we optimize the grating parameters by the rigorous coupled-wave method to obtain the efficiency of each diffraction order, and then use the modal method to explain the physical mechanism of the grating diffraction process.

In this paper, a single-layer five-port transmission grating with a connecting layer under normal incidence is reported and designed. With a duty cycle of $f=0.4$ and a period of $d=2313 \mathrm{~nm}$, the rigorous coupled-wave approach is used to optimize the parameters of the grating. The modal distribution of the grating is analyzed, and the overlap integral of the grating is calculated to explain the diffraction process of the grating. Compared with the single-groove five-port grating, we not only consider the case of TM polarization but also analyze the incident characteristics and process tolerance of the grating.

\section{Numerical design and physical explanation of five-port beam splitters}

Figure 1 is a structural diagram of a five-port transmission grating with a connecting layer, where $h_{1}$ represents the thickness of the grating ridge, $h_{2}$ represents the depth of the connecting layer, and $d$ represents the period of the grating. The grating groove is air, whose refractive index is $n_{1}=1.00$. The grating ridge and the connecting layer are composed of fused silica, and the refractive index is $n_{2}=1.45$. The substrate of the grating is $\mathrm{Ta}_{2} \mathrm{O}_{5}$, and the refractive index is $n_{3}=2.00$. Light with an incident wavelength of $\lambda=800 \mathrm{~nm}$ is incident perpendicularly to the upper surface of the grating. The incident light passes through the grating layer, and the energy of the incident light

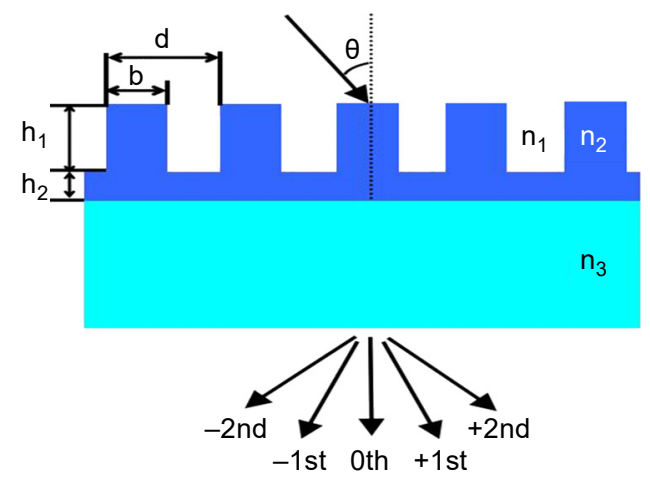

Fig. 1. The schematic diagram of the five-port grating at wavelength of $800 \mathrm{~nm}$ with duty cycle of 0.4 under normal incidence $\theta=0$. 
is diffracted to the 0 th, \pm 1 st and \pm 2 nd orders. Due to the periodic structure of the grating, the efficiency of the +1 st and -1 st orders is equal, the efficiency of the -2 nd and +2 nd orders is equal, so we only consider the transmission efficiency of the 0 th, -1 st and -2 nd orders in the following calculations.

Figure 2 shows the transmission efficiency of a five-port grating under normal incidence $\theta=0$ versus the grating groove depth and the thickness of the connecting layer. The abscissa is the groove depth of the grating $h_{1}$ and the ordinate is the thickness of the grating connecting layer $h_{2}$. It is the contour map, where horizontal and longitudinal coordinates are two thicknesses, and each line in Figs. $2 \mathbf{a}-2 \mathbf{f}$ represents the contour of efficiency. As can be seen from Fig. 2, for TE-polarized light, at $h_{1}=1.35 \mu \mathrm{m}$ and $h_{2}=0.44 \mu \mathrm{m}$, the efficiency of $0 \mathrm{th},-1 \mathrm{st}$, and -2 nd orders is $19.97 \%, 18.83 \%$ and $18.83 \%$, respectively. The total efficiency of the five orders is $95.29 \%$. For the TM-polarized light, when $h_{1}=1.39 \mu \mathrm{m}$ and $h_{2}=1.00 \mu \mathrm{m}$, the efficiency of the 0 th, $-1 \mathrm{st}$ and
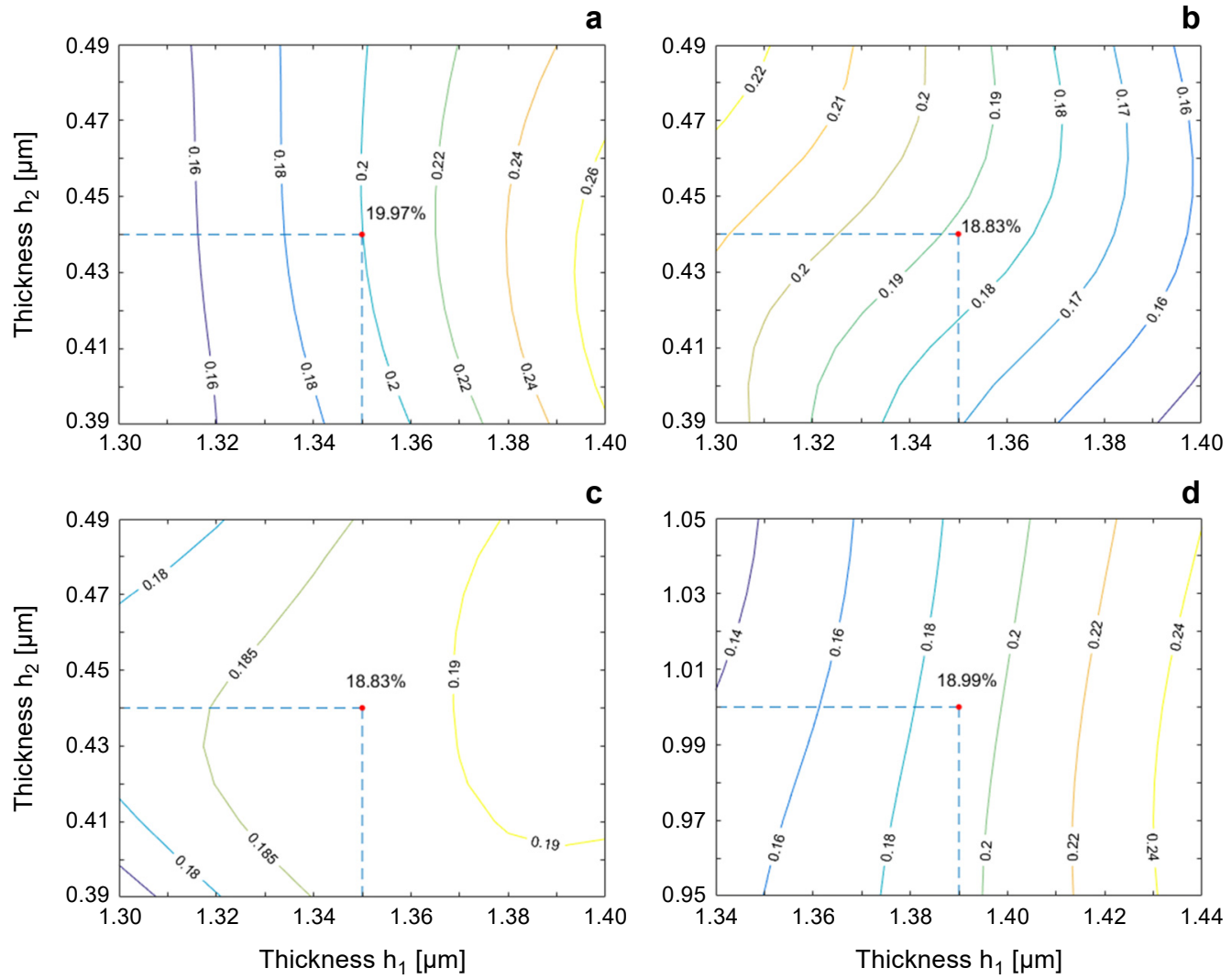

Fig. 2. Efficiencies in different orders for the grating versus the grating groove depth and the thickness of the connecting layer for both two polarizations at wavelength of $800 \mathrm{~nm}$ under normal incidence: the 0th order for TE polarization (a), the -1st order for TE polarization (b), the -2nd order for TE polarization (c), the 0th order for TM polarization (d), the -1 st order for TM polarization (e), the -2 nd order for TM polarization (f). It is the contour map, where horizontal and longitudinal coordinates are two thicknesses, and each line in Figs. 2a-2f represents the contour of efficiency. 

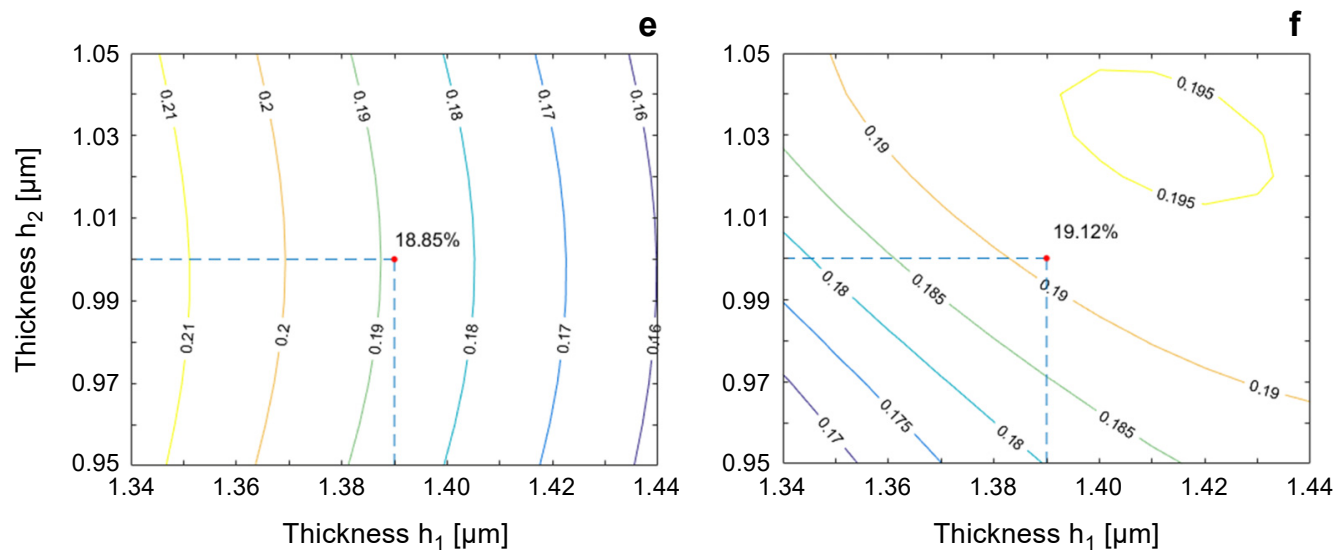

Fig. 2. Continued.

$\mathrm{T} \mathrm{a} \mathrm{b} \mathrm{l} \mathrm{e} 1$. The actual grating profile parameters with the duty cycle of 0.4 under normal incidence.

\begin{tabular}{llllllll}
\hline Polarization & $h_{1}[\mu \mathrm{m}]$ & $h_{2}[\mu \mathrm{m}]$ & $\pm 2 \mathrm{nd}$ & $\pm 1 \mathrm{st}$ & 0 th & Total & Uniformity \\
\hline TE & 1.35 & 0.44 & $18.83 \%$ & $18.83 \%$ & $19.97 \%$ & $95.29 \%$ & $2.93 \%$ \\
TM & 1.39 & 1.00 & $19.12 \%$ & $18.85 \%$ & $18.99 \%$ & $94.93 \%$ & $0.71 \%$ \\
\hline
\end{tabular}

-2 nd orders is $18.99 \%, 18.85 \%$ and $19.12 \%$, respectively. The efficiency of the five orders is $94.93 \%$. The grating structure has better uniformity under TM polarization. Table 1 shows the efficiency and uniformity of the five orders of the grating under two polarizations.

According to the parameters obtained, we use the modal method to explain the physical mechanism of grating diffraction. Figure 3 shows a normalized field diagram of the field distribution of a five-port transmission grating. The $800 \mathrm{~nm}$ of incident light is incident perpendicularly from the surface of the grating, and one can see the energy distribution transmitted by the grating from the bottom. Modal method can be used to explain the diffraction mechanism of the grating. For a single-layer five-port transmission grating, a total of two couplings occur during the diffraction process. The first

a

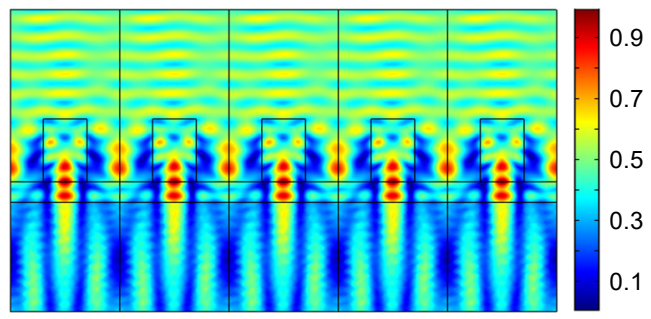

b

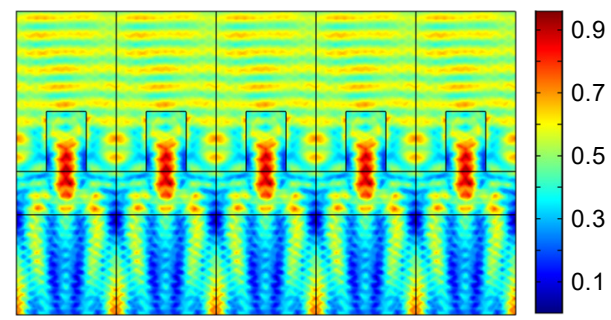

Fig. 3. Normalized field magnitude distribution with five-port beam splitter under normal incidence: TE (a), and TM (b) polarizations. 
$\mathrm{T}$ a b $1 \mathrm{e}$ 2. Effective refractive index of grating for two polarizations.

\begin{tabular}{lll}
\hline & TE & TM \\
\hline Mode 0 & 1.40927 & 1.39882 \\
Mode 1 & 1.28426 & 1.24545 \\
Mode 2 & 1.07756 & 1.04289 \\
Mode 3 & 0.94224 & 0.92478 \\
Mode 4 & 0.88440 & 0.90722 \\
Mode 5 & 0.56625 & 0.55884 \\
\hline
\end{tabular}

$\mathrm{T} \mathrm{a} \mathrm{b} 1$ e 3. Coupling overlap integration between six grating modes and five diffraction orders of an optimized grating parameter at an incident wavelength of $800 \mathrm{~nm}$.

\begin{tabular}{|c|c|c|c|c|c|c|}
\hline & \multicolumn{5}{|c|}{ Mode orders } \\
\hline & & $-2 \mathrm{nd}$ & $-1 \mathrm{st}$ & 0th & $+1 \mathrm{st}$ & $+2 \mathrm{nd}$ \\
\hline \multirow{6}{*}{ 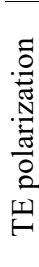 } & Modes 0 & 0.03171 & 0.23971 & 0.45562 & 0.23971 & 0.03171 \\
\hline & Modes 1 & 0.20341 & 0.26176 & 0 & 0.26176 & 0.20341 \\
\hline & Modes 2 & 0.21771 & 0.02963 & 0.24289 & 0.02963 & 0.21771 \\
\hline & Modes 3 & 0.09172 & 0.22672 & 0.28391 & 0.22672 & 0.09172 \\
\hline & Modes 4 & 0.20799 & 0.23082 & 0 & 0.23082 & 0.20799 \\
\hline & Modes 5 & 0.08583 & 0.00266 & 0 & 0.00266 & 0.08583 \\
\hline \multirow{6}{*}{ 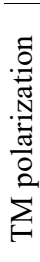 } & Modes 0 & 0.05147 & 0.24258 & 0.40590 & 0.24258 & 0.05147 \\
\hline & Modes 1 & 0.21247 & 0.22143 & 0 & 0.22143 & 0.21247 \\
\hline & Modes 2 & 0.17692 & 0.04303 & 0.32166 & 0.04303 & 0.17692 \\
\hline & Modes 3 & 0.18230 & 0.16310 & 0.09692 & 0.16310 & 0.18230 \\
\hline & Modes 4 & 0.19742 & 0.18574 & 0 & 0.18574 & 0.19742 \\
\hline & Modes 5 & 0.06022 & 0.00075 & 0.00098 & 0.00075 & 0.06022 \\
\hline
\end{tabular}

coupling is the coupling of incident light and grating mode. When the incident light enters the grating region, a first coupling occurs, which triggers the multi-port grating mode. The grating mode propagates through different effective refractive indices $n_{\text {eff }}$ to the exit surface, producing a second coupling. Table 2 shows the effective refractive indices of multiple modes of the grating under two polarizations, which can be obtained by Eqs. (1) to (3) below. The second coupling is the coupling between the grating mode and the diffraction order, wherein the energy exchange in the coupling process can be represented by the overlap integral obtained by Eqs. (4) and (5). Table 3 shows the energy exchange capability under two polarizations.

Grating dispersion equations [28] are: for TE polarization

$$
\begin{aligned}
\cos [ & \left.k_{1}(1-f) d_{\mathrm{TE}}\right] \cos \left(k_{2} f d_{\mathrm{TE}}\right)-\frac{k_{1}^{2}+k_{2}^{2}}{2 k_{1} k_{2}} \sin \left[k_{1}(1-f) d_{\mathrm{TE}}\right] \sin \left(k_{2} f d_{\mathrm{TE}}\right) \\
& =\cos \left(\alpha d_{\mathrm{TE}}\right)
\end{aligned}
$$


and for TM polarization

$$
\begin{aligned}
\cos \left[k_{1}(1-f) d_{\mathrm{TM}}\right] \cos \left(k_{2} f d_{\mathrm{TM}}\right)-\frac{n_{2}^{4} k_{1}^{2}+k_{2}^{2}}{2 n_{2}^{2} k_{1} k_{2}} \sin \left[k_{1}(1-f) d_{\mathrm{TM}}\right] \sin \left(k_{2} f d_{\mathrm{TM}}\right) \\
=\cos \left(\beta d_{\mathrm{TM}}\right)
\end{aligned}
$$

where

$$
\begin{aligned}
& k_{m}=k_{0} \sqrt{n_{m}^{2}-n_{\mathrm{eff}}^{2}} \\
& \alpha=k_{0} \sin \left(\theta_{\mathrm{TE}}\right) \\
& \beta=k_{0} \sin \left(\theta_{\mathrm{TM}}\right) \\
& k_{0}=\frac{2 n_{2} \pi}{\lambda}
\end{aligned}
$$

For TE polarization, the coupling between the exit diffraction order and multiple modes is written as

$$
\left\langle E_{y m}(x) \leftrightarrow u_{m}(x)\right\rangle=\frac{\left|\int_{0}^{d} E_{y m}(x) u_{m}(x) \mathrm{d} x\right|^{2}}{\int_{0}^{d}\left|E_{y m}(x)\right|^{2} \mathrm{~d} x \int_{0}^{d}\left|u_{m}(x)\right|^{2} \mathrm{~d} x}
$$

where $u_{m}(x)$ is the grating mode, and $E_{y m}(x)$ is the diffraction order of the incident electric field. And for TM polarization, the coupling process is written as

$$
\left\langle H_{y w}(x) \leftrightarrow u_{q}(x)\right\rangle=\frac{\left|\int_{0}^{d} H_{y w}(x) u_{q}(x) \mathrm{d} x\right|^{2}}{\int_{0}^{d}\left|H_{y w}(x)\right|^{2} \mathrm{~d} x \int_{0}^{d}\left|u_{q}(x)\right|^{2} \mathrm{~d} x}
$$

where $u_{q}$ stands for grating mode, and $H_{y w}(x)$ is the diffraction order. The left operator \langle\rangle in Eqs. (4) and (5) represents the coupling process between the diffraction order of the grating device and the modes in the grating region. The energy exchange can be described by the overlapping integral on the right side of the formulas, which can explain the physical mechanism of the diffraction process of the grating.

\section{Analysis and discussions}

In the process of optimizing the parameters, the incident wave always is incident perpendicularly. In the actual process, the five-port grating beam splitter has a larger incident angle bandwidth and incident wavelength bandwidth. Therefore, we study the incident angle and incident wavelength of the grating. Figure 4 shows the transmission efficiency 

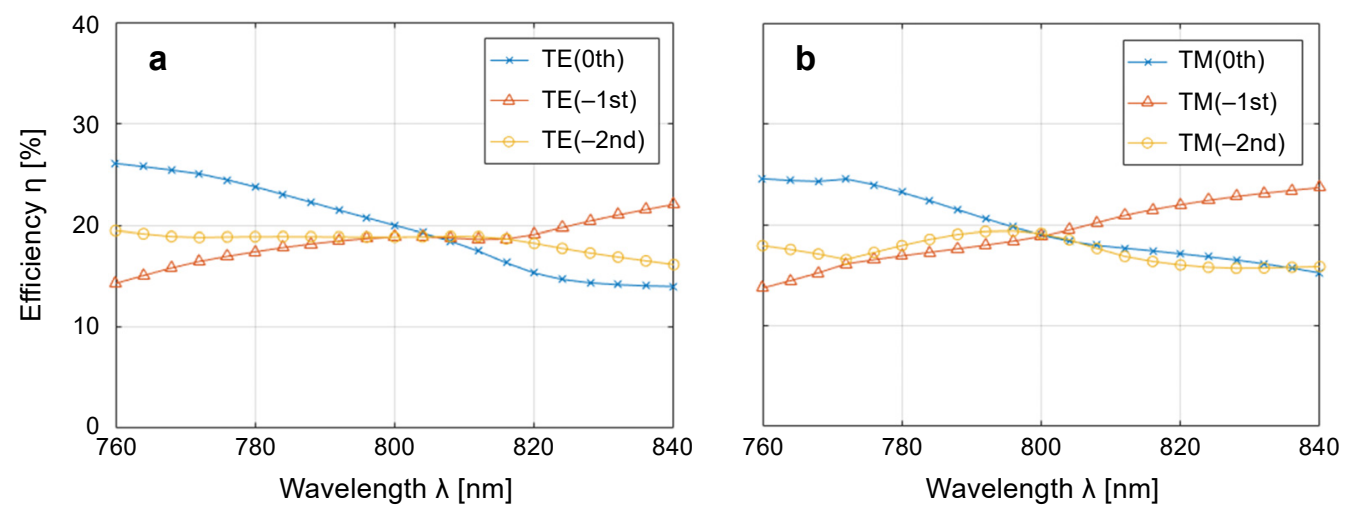

Fig. 4. The efficiency corresponding to the incident wavelength with duty cycle of 0.4 under normal incidence: TE (a), and TM (b) polarizations.
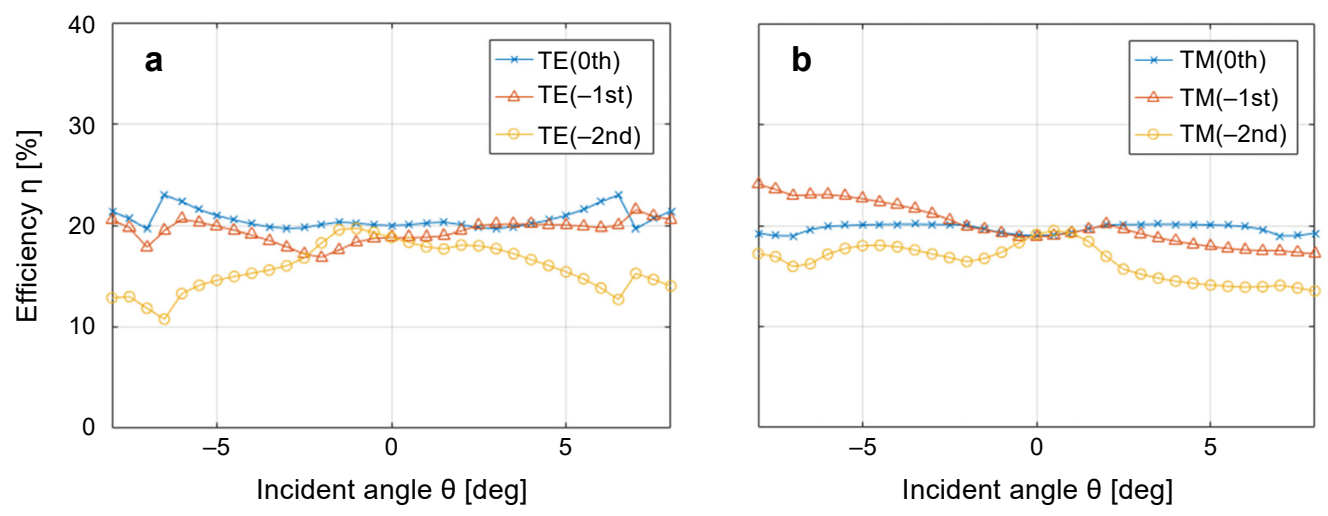

Fig. 5. The efficiency corresponding to the incident angle for TE (a), and TM (b) polarizations.

of five orders versus the incident wavelength. The abscissa indicates the incident wavelength of the grating and the ordinate indicates the diffraction efficiency of the grating. For TE-polarized light, each efficiency of the five orders is greater than $16 \%$ when the wavelength range of the grating is $770-817 \mathrm{~nm}$, where the bandwidth is $47 \mathrm{~nm}$. For TM-polarized light, when the wavelength of the grating is $771-820 \mathrm{~nm}$ and the bandwidth is $49 \mathrm{~nm}$, the respective order of the grating is more than $16 \%$. The grating has a large incident bandwidth under TM polarization. Figure 5 shows the relationship between the incident angle and the grating efficiency. For TE-polarized light, efficiencies of five diffraction orders are greater than $16 \%$ at incident angles ranging from $-3.03^{\circ}$ to $4.54^{\circ}$. For TM-polarized light, the five diffraction orders of the grating are greater than $16 \%$ at incident angles ranging from $-6.60^{\circ}$ to $2.15^{\circ}$. One can see that the TM-polarized grating has a good incident angle bandwidth.

During the actual etching process, the period and duty cycle of the grating may be affected due to some factors in the fabrication process. Therefore, it is necessary to study the manufacturing tolerance of the period and duty cycle of the grating. Figure 6 shows 

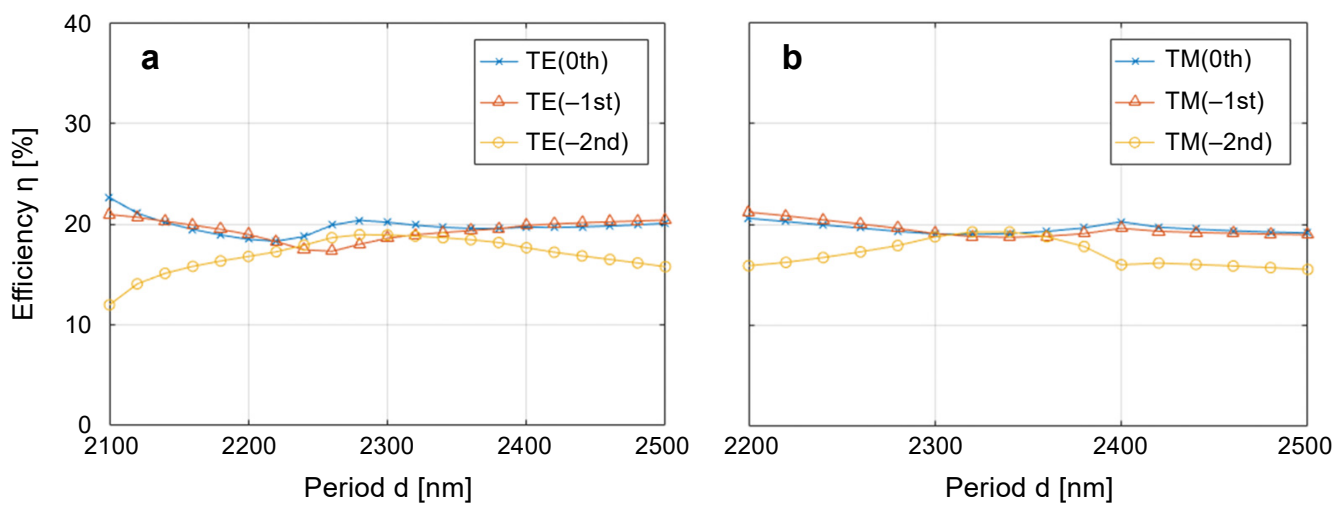

Fig. 6. The diffraction efficiency corresponding to the period: TE (a), and TM (b) polarizations.
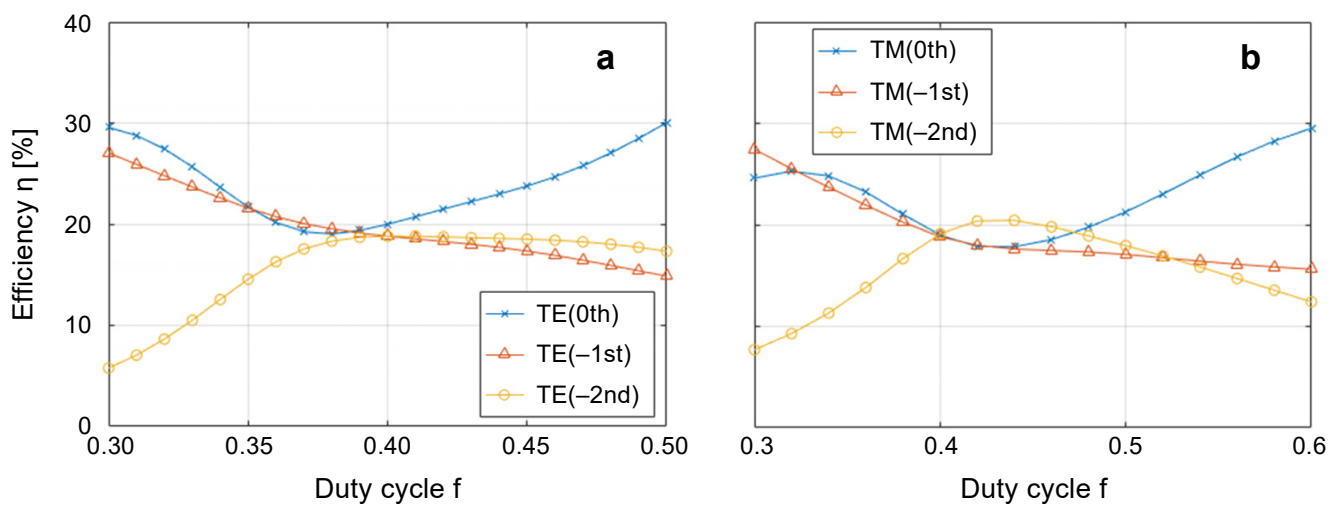

Fig. 7. The diffraction efficiency corresponding to the duty cycle: TE (a), and TM (b) polarizations.

the relationship between the period of the grating and the efficiency of the five orders of the grating. For TE-polarized light, the grating period is in the range of $2168-2487 \mathrm{~nm}$, and the efficiency of the five orders is greater than $16 \%$. For TM-polarized light, the grating period is in the range of 2207-2442 nm, and the efficiency of the five orders is greater than $16 \%$. Figure 7 shows the relationship between the duty cycle of the grating and the efficiency. Under TE polarization, the duty cycle of the grating is from 0.36 to 0.47 , when the efficiency of the five orders is greater than $16 \%$. Under TM polarization, the efficiency of the five orders is greater than $16 \%$ when the duty cycle of the grating is between 0.38 and 0.53 .

\section{Conclusions}

A new single-layer transmissive five-port grating with a connecting layer is designed. The grating beam splitter achieves a better five-port beam splitting effect by adding a connecting layer. We use the rigorous coupled-wave approach to optimize the struc- 
tural parameters of the grating to obtain a good beam splitting effect. Then the modal method is used to calculate the effective refractive index and energy coupling exchange of the excited mode. Based on the optimized parameters, the incident characteristics and process tolerance of the grating are analyzed. The structure shows that the grating has a wide incident bandwidth characteristic under TM polarization and a large period tolerance under TE polarization.

Acknowledgements - This work is supported by the Science and Technology Program of Guangzhou (202002030284, 202007010001, 202002030210) and the Science and Technology Planning Project of Guangdong Province (2020B090924001).

\section{References}

[1] Sun S., Cao Y., Chen C., Fu G., Wang Y., Xu X., A method for measuring the quality parameters of image intensifier based on projecting phase-shifting gratings, Optica Applicata 48(1), 2018, pp. 39-51, DOI: $10.5277 / \mathrm{oa} 180104$.

[2] Mohd Nazal N.A., Lai M., Lim K., Gunawardena D.S., Chong W., Yang H., Ahmad H., Demarcation energy properties of regenerated fiber Bragg grating sensors in few-mode fibers, Optica Applicata 48(2), 2018, pp. 263-271, DOI: 10.5277/oa180209.

[3] Cetin R., AKIN T., Numerical and experimental investigation into LWIR transmission performance of complementary silicon subwavelength antireflection grating (SWARG) structures, Scientific Reports 9, 2019, article 4683, DOI: 10.1038/s41598-019-41107-2.

[4] ZHENG X., WANG Q., ZhANG R., Ma L., LuAN J., Effects of aspect ratio and metal layer thickness on demoulding of metal/polymer bilayer gratings during nanoimprinting, Scientific Reports 8, 2018, article 12720, DOI: 10.1038/s41598-018-31194-y.

[5] Ren Z., Sun Y., Zhang S., Lin Z., Wang C., Tunable narrow band perfect metamaterial absorber based on guided-mode resonance, Modern Physics Letters B 33(16), 2019, article 1950171, DOI: 10.1142/S0217984919501719.

[6] Li Y., Lu P., Zhang C., Ni W., Liu D., Zhang J., Sensing characterization of helical long period fiber grating fabricated by a double-side $\mathrm{CO}_{2}$ laser in single-mode fiber, IEEE Photonics Journal 11(3), 2019, article 6801608, DOI: 10.1109/JPHOT.2019.2913700.

[7] FARTOOKZADEh M., Single-buried-layer reflection-mode metasurfaces for dual-band linear to circular polarization conversion, Modern Physics Letters B 32(23), 2018, article 1850274, DOI: $10.1142 /$ S0217984918502743.

[8] Li D., WANG X., Ling J., YuAN Y., Multiwavelength achromatic microlens through phase compensation based on the subwavelength metallic nanostructures, Optics Communications 445, 2019, pp. 90-95, DOI: 10.1016/j.optcom.2019.04.029.

[9] Wei Liu, Xiaohong Sun, Qingbin Fan, Shuai Wang, Yongle Qi, The investigation of multi-channel splitters and big-bend waveguides based on 2D sunflower-typed photonic crystals, Superlattices and Microstructures 100, 2016, pp. 1291-1295, DOI: 10.1016/j.spmi.2016.11.016.

[10] Wen J., Duan L., FAN W., Influences of pump power and high-order dispersion on dual-pumped silicon-on-insulator micro-ring resonator-based optical frequency combs, Modern Physics Letters B 33(10), 2019, article 1950117, DOI: 10.1142/S0217984919501173.

[11] Li L., Huang T., Zhao X., Wu X., Cheng Z., Highly sensitive SPR sensor based on hybrid coupling between plasmon and photonic mode, IEEE Photonics Technology Letters 30(15), 2018, pp. 1364 -1367, DOI: 10.1109/LPT.2018.2847907.

[12] Ling Q., Gu Z., JiANG X., GAO K., Design of long period fiber grating surrounding refractive index sensor based on mode transition near phase-matching turning point, Optics Communications 439, 2019, pp. 187-192, DOI: 10.1016/j.optcom.2019.01.060. 
[13] Chen Y., Chen L., WANG X., Liu W., A novel metal/dielectric combined grating structure incorporating optically thin plasma metals with the properties of controllably polarization and spectral filtering, Optik 168, 2018, pp. 598-604, DOI: 10.1016/j.ijleo.2018.04.124.

[14] Feng Y., Liu Y., Shi Y., Teng J., Tunable plasmonic filter based on graphene-layered waveguide, Modern Physics Letters B 32(8), 2018, article 1850110, DOI: 10.1142/S0217984918501105.

[15] BAi B., Deng Q., Zhou Z., Plasmonic-assisted polarization beam splitter based on bent directional coupling, IEEE Photonics Technology Letters 29(7), 2017, pp. 599-602, DOI: 10.1109/LPT.2017. 2675448.

[16] Xiong J., Yu Y., Yang W., Sun C., Zhang X., Crosstalk suppressed high efficient mode-selective four-wave mixing through tailoring waveguide geometry, IEEE Photonics Journal 11(1), 2019, article 6600408, DOI: 10.1109/JPHOT.2019.2891335.

[17] Ren J., Sun X., WANG S., A narrowband filter based on 2D 8-fold photonic quasicrystal, Superlattices and Microstructures 116, 2018, pp. 221-226, DOI: 10.1016/j.spmi.2018.01.017.

[18] Wang S., Wu H., Zhang M., Dai D., A 32-channel hybrid wavelength-/mode-division (de)multiplexer on silicon, IEEE Photonics Technology Letters 30(13), 2018, pp. 1194-1197, DOI: 10.1109/LPT.2018. 2839533.

[19] Luo H., Cheng Y., Design of an ultrabroadband visible metamaterial absorber based on three-dimensional metallic nanostructures, Modern Physics Letters B 31(25), 2017, article 1750231, DOI: 10.1142/S0217984917502311.

[20] CAO J., YAnG G., WANG J., GaO S., Lu Y., Sun R., YAn P., Enhanced optical absorption of monolayer $W_{2}$ using Ag nanograting and distributed Bragg reflector structures, Superlattices and Microstructures 112, 2017, pp. 218-223, DOI: 10.1016/j.spmi.2017.09.030.

[21] Ren Z., Sun Y., Zhang S., Zhang K., Hu J., Lin Z., Active optical switches based on polarizationtuned guided-mode resonance filters for optical communication, Optics Communications 426, 2018, pp. 383-387, DOI: 10.1016/j.optcom.2018.05.051.

[22] Xu F., Zhu J., Fan S., QI Y., Control of slow light in three- and four-level graphene nanostructures, Modern Physics Letters B 33(20), 2019, article 1950226, DOI: 10.1142/S0217984919502269.

[23] Caо H., Zhou C., Feng J., Lu P., Ma J., Design and fabrication of a polarization-independent wideband transmission fused-silica grating, Applied Optics 49(21), 2010, pp. 4108-4112, DOI: 10.1364/ AO.49.004108.

[24] Feng J., Zhou C., Wang B., Zheng J., Jia W., Cao H., Lv P., Three-port beam splitter of a binary fused-silica grating, Applied Optics 47(35), 2008, pp. 6638-6643, DOI: 10.1364/AO.47.006638.

[25] XIANG C., ZHOU C., JIA W., Wu J., Five-port beam splitter of a single-groove grating, Chinese Optics Letters 16(7), 2018, article 070501, DOI: 10.1364/COL.16.070501.

[26] Moharam M.G., Pommet D.A., Grann E.B., GaYlord T.K., Stable implementation of the rigorous coupled-wave analysis for surface-relief gratings: enhanced transmittance matrix approach, Journal of the Optical Society of America A 12(5), 1995, pp. 1077-1086, DOI: 10.1364/JOSAA.12.001077.

[27] Botten I.C., Craig M.S., McPhedran R.C., Adams J.L., Andrewartha J.R., The dielectric lamellar diffraction grating, Optica Acta: International Journal of Optics 28(3), 1981, pp. 413-428, DOI: $10.1080 / 713820571$.

[28] Zнао H., Reverse design for diffraction gratings by modal method, Optik 157, 2018, pp. 621-627, DOI: $10.1016 /$ j.ijleo.2017.11.139.

Received September 23, 2019 in revised form November 13, 2019 